\title{
A Novel Technique for the Metrology Calibration of a Fourier Transform Spectrometer
}

\author{
Locke D. Spencer and David A. Naylor \\ Department of Physics, University of Lethbridge, \\ 4401 University Drive, Lethbridge, AB, CA, T1K $3 M 4$ \\ Locke.Spencer@uleth.ca
}

\begin{abstract}
A method is presented for using a Fourier transform spectrometer (FTS) to calibrate the metrology of a second FTS. This technique is particularly useful when the second FTS is inaccessible such as inside a cryostat.

(c) 2006 Optical Society of America
\end{abstract}

OCIS codes: (120.3180) Interferometry; (120.6200) Spectrometers and spectroscopic instrumentation.

\section{Introduction}

When an FTS is operated at cryogenic temperatures there are instances in which it is difficult to verify the performance of the metrology system. The FTS of the Herschel/SPIRE spectrometer is one such example[1]. In this case the optical path difference (OPD) is measured with a linear Heidenhain encoder and a redundant backup system[2]. These units are calibrated at room temperature and it is then assumed that, given the low expansion coefficients of the materials used in the construction of the encoders, their performance will remain unchanged when cooled to liquid Heluium temperatures. The SPIRE proto-flight model (PFM) verification tests are performed at the Rutherford Appleton Laboratory (RAL). This test facility houses a number of pieces of test instrumentation external to the SPIRE cryostat. One of these instruments is a test FTS (TFTS) whose primary purpose is to measure the spectral characteristics of the SPIRE photometer[3]. During recent PFM tests the TFTS was also used in conjunction with the SPIRE spectrometer. Analysis has shown that the dual FTS configuration can be used to calibrate the metrology of the SPIRE spectrometer under near flight conditions when it is enclosed within the SPIRE test cryostat.

\section{Theory}

The irradiance received by a detector is related to the electric field as follows:

$$
I=\frac{c \varepsilon_{o}}{2}\left|{\overrightarrow{E_{o}}}^{*} \overrightarrow{E_{o}}\right| \quad\left[W / m^{2}\right],
$$

where $c$ is the speed of light, $\varepsilon_{o}$ is the permittivity of free space, and $\overrightarrow{E_{o}}$ is the electric field at the detector. In the case of a two beam interferometer, for each input electric field there are two output electric fields[4]. The measured irradiance can then be expressed as:

$$
I\left(z_{\mathrm{OPD}}\right)=c \varepsilon_{o}\left|\overrightarrow{E_{o}(\sigma)}\right|^{2} r^{2} t^{2}+c \varepsilon_{o}\left|\overrightarrow{E_{o}(\sigma)}\right|^{2} r^{2} t^{2} \cos \left(k z_{\mathrm{OPD}}\right),
$$




\section{FTuB4.pdf}

where $k=2 \pi \sigma$ is the magnitude of the wave vector, $\sigma$ is the wavenumber $\left(\mathrm{cm}^{-1}\right)$, and $z_{\mathrm{OPD}}$ $(\mathrm{cm})$ is the optical path difference, and $r$ and $t$ are the reflectance and transmittance of the beam divider[4].

The technique we discuss in this paper involves two FTSs placed in series as shown in figure 1. Radiation incident at the input of the TFTS is directed towards the SPIRE FTS and then output towards the SPIRE detectors. An electric field at the input of the TFTS produces two electric fields its the output. These two electric fields, which are now inputs to the SPIRE FTS, produce four electric fields at the SPIRE output. The irradiance at the SPIRE detectors is then the superposition of four electric fields; this irradiance may be expressed as:

$$
\begin{aligned}
& I_{\text {dual FTS }}\left(z_{\mathrm{OPD} 1}, z_{\mathrm{OPD} 2}\right)=c \varepsilon_{o}\left|E_{o}(\sigma)\right|^{2} t_{1}^{2} r_{1}^{2} t_{2}^{2} r_{2}^{2}\left\{\quad 2+2 \cos \left(k z_{\mathrm{OPD} 2}\right)\right. \\
& +2 \cos \left(k z_{\mathrm{OPD} 1}\right) \\
& +\cos \left(k z_{\mathrm{OPD} 1}-k z_{\mathrm{OPD} 2}\right) \\
& \left.+\cos \left(k z_{\mathrm{OPD} 1}+k z_{\mathrm{OPD} 2}\right)\right\} \text {, }
\end{aligned}
$$

where the subscripts 1 and 2 refer to the TFTS and the SPIRE FTS respectively. By inspection it can be seen that if the SPIRE FTS is held at a fixed position while the TFTS is scanned, there will be four interference patterns observed corresponding to each of the terms in equation (3). Two of these terms, $c \varepsilon_{o} E_{o}^{2} r_{1}^{2} t_{1}^{2}\left(r_{2}^{2} t_{2}^{2}\right) \cos \left(k z_{\mathrm{OPD} 1} \pm k z_{\mathrm{OPD} 2}\right)$, are secondary interference patterns not observed with just a single FTS.

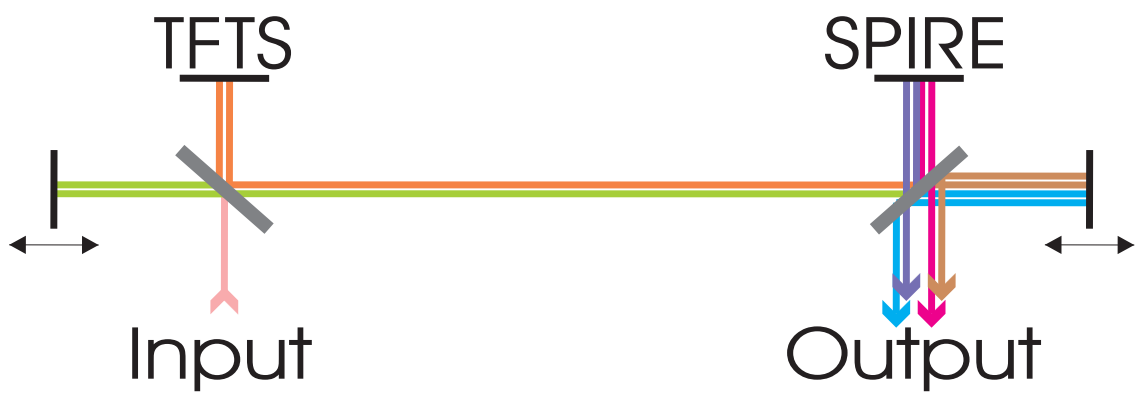

Fig. 1. Electric field propagation through dual FTS configuration.

For a broad-band source, the interference pattern is narrow in the spatial domain. In equation (3) the three cosine terms constitute three unique positions of constructive interference at $z_{\mathrm{OPD} 1}=$ 0 and $\pm z_{\mathrm{OPD} 2}$. (Note that equation (3) simplifies to equation (2) for $z_{\mathrm{OPD} 2}=0$.) Since the observed interferogram signal for a broadband source is a local maximum, it is the region of highest signal-to-noise ratio within the interferogram and thus can be analyzed to determine the location of ZPD to high precision, the same holds for $z_{\mathrm{OPD} 2}$. Figure 2 illustrates the observed dual FTS signal for various values of $z_{\mathrm{OPD} 2}$ and demonstrates how an external FTS, whose metrology is easily calibrated outside of the cryostat, can be used to test the metrology of an inaccessible FTS.

\section{Results}

The results presented in this section demonstrate the dual FTS technique and were obtained during the SPIRE PFM tests in May 2006. Approximately 35 scans were recorded in which the TFTS $\left(z_{\mathrm{OPD} 1}\right)$ was scanned while the SPIRE FTS $\left(z_{\mathrm{OPD} 2}\right)$ was held constant. The results are 


\section{FTuB4.pdf}
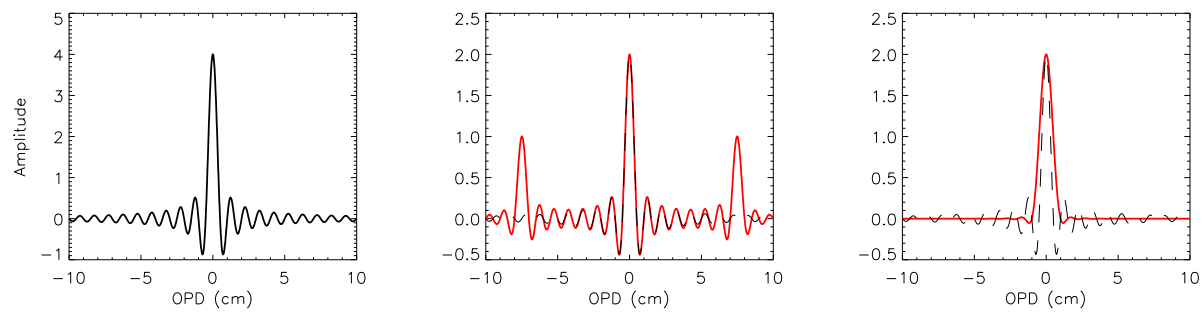

Fig. 2. A comparison of dual FTS methods using $\mathrm{OPD}_{\text {calib }}=0 \mathrm{~cm}$ (left), $7.5 \mathrm{~cm}$ (centre) and $0.5 \mathrm{~cm}$ (right). The interference pattern shown is a sinc function which results from a rectangular spectrum. The dashed line shows the interference pattern that would be observed if only one FTS were used in the observation (scaled).

shown in figure 3 where the left plot shows one of the recorded interferograms as a function of $z_{\mathrm{OPD} 1}$ and the right plot shows the calculated values of $z_{\mathrm{OPD} 2}$. From parabolic fitting of the three local maxima in the interferogram signal, the SPIRE stage position $\left(z_{\mathrm{OPD} 2}\right)$ was determined to be $16.298 \pm 0.001 \mathrm{~mm}$. Since the SPIRE FTS is of the Mach-Zehnder design[1], and has a multiplying factor of 4 in OPD, the mechanical position of the SPIRE stage is determined to an accuracy $\pm 0.25 \mu \mathrm{m}$ under near flight conditions.
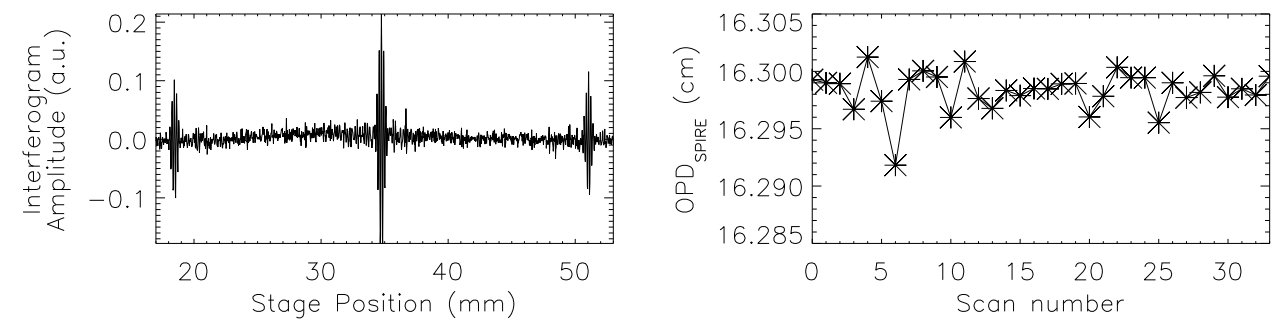

Fig. 3. Measurements of the dual FTS calibration of the SPIRE spectrometer.

\section{Conclusions}

A novel technique for the non-invasive metrology calibration of an FTS has been described. This technique has a distinct advantage in that it uses the same detectors used to measure infrared radiation to verify the metrology of the spectrometer. For imaging FTSs, the variation in OPD for off-axis pixels, i.e. the obliquity factor, can also be studied.

\section{References}

1. M. J. Griffin and B. M. Swinyard, "Herschel-SPIRE: design, performance, and scientific capabilities," SPIE Proceedings, Space Telescopes and Instrumentation I:Optical,Infrared, and Millimeter, Vol. 6255 (2006).

2. D. Griffin, M. J. Griffin, and B. M. Swinyard, "SPIRE Design Description," Rutherford Appleton Laboratory Technical Note, SPIRE-RAL-PRJ-000620, ver. 2.0, May 2003.

3. L. D. Spencer, D. A. Naylor, B. M. Swinyard, A. A. Aramburu, T. R. Fulton, T. L. Lim, S. D. Ronayette, and I. S. Schofield, "A Fourier Transform Spectrometer for Ground Testing of the Herschel/SPIRE Instrument," SPIE Proceedings: Astronomical Telescopes and Instrumentation, Vol. 5487, pp. 501-512, June 2004.

4. R. J. Bell, "Introductory Fourier Transform Spectroscopy,” Academic Press, New York (1972). 